Horizons philosophiques

\title{
Vers une éthique de la désacralisation de la vie
}

\section{Yvon Provençal}

Volume 4, numéro 2, printemps 1994

Médecines impossibles?

URI : https://id.erudit.org/iderudit/800950ar

DOI : https://doi.org/10.7202/800950ar

Aller au sommaire du numéro

\section{Éditeur(s)}

Collège Édouard-Montpetit

\section{ISSN}

1181-9227 (imprimé)

1920-2954 (numérique)

Découvrir la revue

Citer cet article

Provençal, Y. (1994). Vers une éthique de la désacralisation de la vie. Horizons philosophiques, 4(2), 81-109. https://doi.org/10.7202/800950ar d'utilisation que vous pouvez consulter en ligne.

https://apropos.erudit.org/fr/usagers/politique-dutilisation/ 


\section{VERS UNE ÉTHIQUE DE LA DÉSACRALISATION DE LA VIE}

\section{Critique de l'argumentation basée sur le caractère sacré de la vie dans les cas de l'avortement, de l'euthanasie et du suicide.}

On admet très souvent, sans critique, le «caractère sacré» de la vie humaine. On se base sur ce présupposé pour refuser le droit à l'avortement ou à l'euthanasie par exemple, ou alors on accorde ce droit mais avec mauvaise conscience. Habituellement les tenants du respect de la vie présenté comme inconditionnel ont beau jeu du point de vue éthique. Ils disposent, sinon de doctrines ou de systèmes persuasifs et sans failles, du moins d'une vision plus globale où la morale et les considérations historiques se conjuguent pour asseoir des principes sincères et solides. En face, ceux qui défendent le droit à l'avortement libre ou à l'euthanasie active paraissent bien opportunistes. Leurs arguments semblent être fonction de la conjoncture actuelle particulière. Ils prennent la forme de revendications individuelles étroites et à courte vue.

Dans la plupart des pays occidentaux, on a entrepris de décriminaliser et, dans certains cas, de déjudiciariser l'avortement et l'euthanasie. II reste à déculpabiliser. On a ainsi commencé à reconnaître de factole caractère relatif du respect de la vie en tant que telle. Cependant, on a beaucoup de difficultés à admettre que cette reconnaissance constitue un apport positif au crédit de la sensibilité morale contemporaine.

Nous entendons montrer, dans cet article, qu'il est possible de concevoir une éthique du droit au refus de la vie dont les bases sont solides et convaincantes, qui s'enracine dans l'histoire et qui, au surplus, tient vraiment compte de la personne humaine. Cependant, on se heurte en ces matières à des résistances profondes. II nous faudra prendre une approche inhabituelle. II nous faudra comprendre à quoi tiennent exactement ces résistances. Pour ce faire nous examinerons, en une certaine 
perspective historique, ce qui fait essentiellement la modernité de cette problématique. L'utilisation du mot «modernité», ici, signifie que nous prenons conscience que quelque chose est advenu dans les conceptions morales et dans la sensibilité morale au cours des deux derniers siècles de l'histoire. Ce quelque chose est inouï. II consiste, entre autres, dans le droit qui est reconnu à l'instance individuelle de trouver en ellemême sa propre justification, son propre sens. Dès lors, il est problématique d'opposer aux choix libres des individus quoi que ce soit d'intangible ou de "sacré», quand ce serait la vie même. Notre approche consiste à étudier en elle-même l'argumentation des tenants du caractère sacré ou intouchable de la vie et de la mettre en rapport avec d'autres argumentations qui ont eu cours dans l'histoire des conceptions morales, d'autres argumentations d'ailleurs largement oubliées (même par les moralistes actuels, semble-t-il). Ainsi, nous dégagerons des rapports de correspondance entre conceptions appartenant à différentes époques. Plus particulièrement, nous examinerons comment la conception classique de l'autorité paternelle, laquelle était, aux dix-septième et dix-huitième siècles, considérée comme sacrée et incontournable, soutenait une argumentation qui, par plusieurs points, correspond de façon frappante à l'argumentation des tenants actuels des positions morales restrictives en matière d'avortement ou d'euthanasie. Si bien que nous pourrons nous appuyer sur la forme de la réfutation de cette vieille argumentation - et de la sensibilité morale qui l'accompagnait - pour faire de même avec cette autre argumentation, qui a cours actuellement. Cette mise en correspondance sera de nature à éclairer d'autres aspects de la problématique générale concernant le respect de la vie. Nous montrerons comment chaque point essentiel de l'argumentation moderne (basée sur le caractère sacré de la vie) correspond formellement à un point précis de l'argumentation classique (reposant sur le principe du caractère sacré de l'autorité paternelle). 


\section{Arguments existant contre le caractère sacré de la vie.}

Une certaine argumentation est aujourd'hui mise de l'avant (notamment par les tenants du libre choix en matière d'avortement ou d'euthanasie) contre le caractère intouchable de la vie. Examinons-la avant d'identifier les raisons profondes pour lesquelles elle se heurte à un mur d'incompréhension et d'intolérance, et pour lesquelles elle ne se produit le plus souvent que dans le manque d'assurance et la mauvaise conscience.

Le respect de la vie en est venu à signifier, lorsqu'on l'entend en un sens fort, le respect de la personne humaine. L'expression dénote d'abord la dimension biologique de l'existence de la personne, mais cela se comprend de telle manière que la "vie» apparaît comme la condition essentielle de toute existence réelle. Dans la Déclaration universelle des droits humains, le droit à la vie domine tous les autres. On considère le principe du respect de la vie comme le premier principe de toute société humaine. Cela est vrai du moins au niveau de l'intention. Dans les faits, c'est différent. On peut remarquer qu'en Occident, on vit dangereusement et qu'on le fait souvent dans un esprit d'amusement. Or même les plus ardents défenseurs du respect de la vie ne songent guère à faire interdire les sports dangereux (alpinisme, plongée sous-marine, etc.), ni, bien sûr, les activités normales ou les métiers qui comportent des risques notables (conduite automobile, travaux de construction, etc.).

Des calculs précis permettent d'évaluer le coût d'une vie humaine dans nos sociétés. Cela entre dans les considérations sur les coûts de la santé et sur l'économie en général. En pratique, on se trouve à accepter qu'une vie individuelle soit contrebalancée par une valeur monétaire. Ainsi admet-on implicitement, mais inévitablement, qu'on ne veuille débourser au-delà de ce qu'on fait pour améliorer la sécurité générale ou prolonger des vies.

II arrive souvent, par ailleurs, que l'on relie le caractère sacré de la vie au courant historique de la pensée chrétienne. Ainsi on pense arguer que le respect de la vie est une tradition 
morale aussi essentielle à l'Occident que sa religion même. Or ce point est discutable. Le respect absolu de la vie a des racines culturelles qui remontent bien au-delà du christianisme.

Certains auteurs modernes concluent à la nécessité de désacraliser la vie. Le professeur de sciences religieuses Éric Volant ${ }^{1}$ estime que nous devons nous prémunir contre toute survalorisation de la vie, laquelle est, selon lui, entretenue idéologiquement dans le monde actuel. Ce serait alors une idéologie en perte de vitesse. Les débats actuels autour de la question de l'avortement démontreraient qu'est apparue une conception du respect de la vie individuelle qui s'oppose au respect de la Vie, conçue de façon abstraite. Le docteur Robert de Montvalon ${ }^{2}$ affirme que la peur qu'inspire le sujet de l'avortement, loin de prouver que l'on respecte la vie, montre que l'on refuse de respecter effectivement les vivants autant qu'on le pourrait.

Cependant, ces arguments ne permettent pas de faire avancer beaucoup le débat, dans la mesure où ils ne font pas comprendre ce qui fait la force de la position adverse, en quoi celle-ci s'enracine et où elle prend son pouvoir de conviction. Ces points doivent être tirés au clair si nous voulons effectuer un véritable dépassement éthique.

\section{Pourquoi la vie est-elle considérée comme sacrée?}

En première analyse, "sacré» veut dire «intouchable» ou "dont le contact est prohibé». L'étymologie du mot renvoie au latin sacer, dont le sens original, difficilement traduisible, est analogue à celui du mot polynésien tabou. Celui-ci signifie notamment: inquiétant, dangereux, interdit, impur.

Les significations du «sacré» plongent loin leurs racines dans l'univers de l'humain. Elles sont relatives aux cultures et aux époques. Toutefois, les prohibitions du type tabou paraissent généralement naturelles - c'est-à-dire nécessaires - à ceux qui vivent sous leur empire.

1. É. Volant, Les Suicidés (ouvrage collectif), Montréal, éd. Fides, 1985, p. 13

2. R. de Montvalon, Problèmes éthiques de l'avortement (ouvrage collectif), Toulouse, éd. Édouard Privat, 1973, p. 172 
Il est frappant que ceux qui affirmentle caractère "sacré» de la vie utilisent un mode d'expression déphasé en regard du langage de l'intellectuel contemporain. Comme ce qui connote une foi religieuse, le mot "sacré" est alors utilisé de façon à accompagner une attitude sérieuse, souvent teintée d'intolérance ou d'absolutisme. Par contre, les tenants des sciences humaines paraissent souvent très relativistes. Ils n'utilisent guère le mot "sacré" que dans un sens extérieur et dans un esprit studieux.

L'ethnologue sait depuis longtemps que la vie des sociétés dites primitives est dominée par trois grands interdits, liés entre eux :

- la prohibition de l'inceste (c'est-à-dire du mariage intratribal),

- la prohibition de l'homicide (à l'intérieur de sa tribu),

- la prohibition de manger le totem (associé à sa tribu).

Le psychologue ou le psychanalyste interprètent ces interdits de diverses manières. Freud y a vu les symptômes d'une névrose obsessionnelle ("maladie du tabou"). Les obsédés disent avoir un pressentiment indéfinissable que la violation serait une cause de préjudice grave pour des personnes de leur entourage. Freud a retenu des observations anthropologiques que quiconque viole le tabou devient tabou lui-même. Ce faisant, il se trouve à se convaincre lui-même qu'il est atteint d'un mal incurable et mortel.

Le malfaiteur innocent qui a, par exemple, mangé de la chair d'un animal prohibé, tombe dans un état de profonde dépression, attend la mort et finit réellement par mourir3.

Quelle que soit l'exactitude anthropologique dans le détail de ces observations freudiennes, une chose est claire. Si Freud, au lieu de prendre l'exemple de la manducation du totem, avait pris l'exemple de l'homicide, son illustration n'aurait

3. S. Freud, Totem et tabou. Interprétation par la psychanalyse de la vie sociale des peuples primitifs, Paris, Payot, 1988, page 32. 
pas produit le même effet. Dans la société occidentale moderne, tout se passe comme si, parmi les trois grands interdits primitifs, ne subsistait plus que la prohibition de l'homicide, ou d'actes apparentés tels que l'avortement ou l'aide à l'euthanasie. On considère encore comme normal, dans ce cas, que l'individu qui commet de tels actes se comporte comme s'il avait violé un tabou et devienne lui-même tabou. Il se met en quelque sorte au ban de l'humanité (ici confondue avec la «tribu»). Il est par le fait même inquiétant, impur et dangereux.

L'attitude de Freud lui-même est révélatrice. Ce qui le frappe ce n'est pas la persistance du tabou dans le monde moderne. Il prend plutôt le point de vue inverse. Ce ne sont pas les modernes qui ont conservé un restant de mentalité primitive ou archaïque, mais bien plutôt ce sont les peuples dits primitifs qui l'étonnent d'avoir déjà conçu une telle interdiction. Freud écrit :

Ceux qui étaient portés à attribuer aux peuples primitifs une cruauté impitoyable et sans merci à l'égard de leurs ennemis, apprendront avec d'autant plus d'intérêt que chez eux aussi le meurtre d'un homme ne pouvait être accompli sans l'observance de certaines prescriptions qui font partie des coutumes tabous 4 .

II ajoute de façon significative :

On dirait que ces primitifs connaissaient déjà le commandement: tu ne tueras point...5.

Nous avons un début d'explication anthropologique pour le fait que la vie est considérée comme sacréê. On trouve l'origine de cette conception dans les grands interdits, ou tabous, des sociétés dites primitives. Le psychologue allemand W. Wundt estimait déjà, au dix-neuvième siècle, que le tabou est le motif profond de nos prescriptions morales et de nos lois. Cependant, le cas du respect de la vie humaine, et le rôle que ce principe joue encore dans nos attitudes morales et nos législations, 
posent un problème particulier. On continue dans ce cas de mettre de l'avant la sacralité. Ce seul fait devrait mériter plus d'attention qu'il n'en reçoit généralement. Certes, on alléguerait que la perte d'une vie humaine est en soi plus grave que le fait de manger une nourriture prohibée pour des motifs religieux ou même que d'avoir des rapports sexuels avec des membres de sa propre famille. Une telle remarque ne résout rien. Elle ne fait que confirmer le problème. C'est-à-dire que sa fausse évidence confirme le problème. Pourquoi donc le refus de la vie en général - y compris les cas d'euthanasie et d'avortement entre autres - est-il vu comme une violation d'un interdit fondamental?

Freud remarque bien la survivance moderne de certains tabous et il amorce là-dessus une formulation problématique. II constate que, de nos jours encore, le bourreau professionnel est soumis à l'isolement temporaire ou permanent. En outre, il va jusqu'à interpréter l'impératif catégorique de Kant comme une survivance du tabou. II explique que ce principe, si important pour toute la conception de l'éthique moderne, a une fonction analogue à celle du tabou et agit à peu près comme le tabou, même si, à la différence de ce dernier, il met en œuvre une motivation ${ }^{6}$ consciente et prétend être un gage d'autonomie individuelle. Un philosophe aurait du mal, sans doute, à admettre une telle interprétation; car il ferait une distinction essentielle entre une attitude irrationnelle et une attitude basée sur un principe formel bien réfléchi qui, au surplus, a joué un rôle capital dans toute la pensée éthique moderne.

Considérer le caractère sacré de la vie comme une survivance de tabous archaïques n'est d'ailleurs pas satisfaisant. Certains faits de l'histoire montrent que la situation se présente de façon plus complexe. La conception moderne du respect de la vie se recommande volontiers d'Hippocrate, dont le serment célèbre, encore invoqué aujourd'hui par des médecins et des moralistes, met de l'avant le caractère absolu du respect de la vie humaine. Toutefois, les conceptions de l'An-

6. Idem, préface, page 6 . 
tiquité ne vont pas toutes dans le même sens. II s'en faut de beaucoup. Par exemple, les Lacédémoniens considéraient comme parfaitement normal de précipiter dans le Taygète les enfants difformes, qui seraient incapables de devenir de bons guerriers. Qu'on pense aussi aux conceptions des Romains. Le paterfamilias avait un droit effectif de vie et de mort sur sa progéniture. De plus, les jeux de cirques donnaient lieu à des actes sanglants où le respect de la vie n'avait pas de place. Deux penseurs de l'époque, Cicéron et Sénèque, ont d'ailleurs critiqué ces jeux à cause de leur indignité et nullement au nom du respect de la vie.

\section{Une question de liberté plutôt que de vie ou de mort.}

Les conceptions modernes en matière de morale sont en rupture avec celles du passé. Cependant, cette rupture ne consiste nullement en une reconnaissance nouvelle d'un quelconque caractère sacré de la vie. Bien au contraire, puisque ce caractère sacré découle du vieux caractère tabou entretenu dans les sociétés dites primitives. La rupture moderne consiste bien plutôt dans l'apparition, inouie dans toute l'histoire, de la notion de respect des choix individuels qui sont faits en vue de l'accomplissement personnel. Cette affirmation n'est d'ailleurs pas le produit d'une simple vue de l'esprit. Elle découle d'un état de fait qui existe réellement, bien que de façon souvent implicite.

La reconnaissance de la liberté individuelle est le fait qui tranche vraiment avec le passé, plutôt que le respect du caractère sacré de la vie, et cette reconnaissance commande tout un faisceau de conceptions nouvelles qui sont caractéristiques de l'espritéthique de la modernité. Nous pouvons illustrer celui-ci en examinant en particulier quelques attitudes nouvelles. Par exemple un des traits de l'esprit éthique moderne est l'intolérance nouvelle vis-à-vis de la torture, quelle que soit l'utilité de cette dernière. La plupart des polices du monde savent que la torture peut être très efficace pour solutionner certains problèmes de sécurité. En particulier, le rendement de la torture en vies humaines est notoire. Le voyageur conféren- 
cier Jacques Chegaray rapporte les propos suivants d'un officier français, lors de la guerre d'Indochine, sur la torture qu'il ordonnait d'infliger aux prisonniers : «c'est la seule façon de les faire parler... Dix minutes d'interrogatoire à la machine (produisant des chocs électriques) et vous obtenez un renseignement sur un champ de mines piégées ou sur une embuscade. Et cela sauve la vie à dix de mes hommes...". Or toute la conception moderne du respect de la personne s'oppose radicalement à la torture qui, sans doute pour la première fois dans l'histoire de l'humanité, est considérée comme universellement condamnable. Le caractère sacré de la vie n'est donc pas, en fait, le facteur déterminant des attitudes éthiques.

Donnons un autre exemple significatif de réprobation commandée par une sensibilité morale nouvelle. II s'agit de la réaction provoquée par un auteur qui est en faveur de l'euthanasie mais pour des raisons complètement inacceptables à cette sensibilité moderne qui se trouve en rupture avec le passé. Dans L'Homme, cet inconnu (1935), Alexis Carrel s'est prononcé en faveur de l'euthanasie, mais il s'agit d'une euthanasie appliquée de force aux criminels. II écrit là : "C'est la qualité des êtres humains qui importe et non leur quantité..." Notons bien ici un point important pour éviter un type de confusion très répandu : ce propos de Carrel est jugé en fait inacceptable non parce qu'il prend la défense de l'euthanasie en tant que telle, mais bien plutôt parce qu'il se fait partisan d'une forme de législation qui va à l'encontre de l'autonomie individuelle. C'est d'ailleurs toute la différence - et elle est essentielle - entre l'euthanasie coercitive et l'euthanasie autonome. La première s'attaque à l'individu en lui interdisant de vivre pour des considérations de rendement collectif. La deuxième reflète l'orientation autonome de l'individu à l'égard de sa propre vie. Alors, de toute évidence, ce n'est pas son manque de respect pour la vie humaine que l'on doit reprocher à Carrel si on veut être en accord avec la tendance de fond de la sensibilité morale apparue nouvellement dans la modernité, mais bien son dédain pour la liberté des choix des individus en ce qui concerne leur propre vie. En ce sens, nous pouvons dire 
que cette orientation contestable de Carrel est nettement plus en accord avec ceux qui continuent de faire passer le caractère sacré de la vie avant l'autonomie individuelle qu'avec leurs opposants.

Un des arguments forts de ceux qui s'opposent à toute libéralisation des choix sur les questions de vie ou de mort est que le respect absolu de la vie constitue une conquête de l'histoire humaine et que tout recul là-dessus équivaut à une régression vers la barbarie. On compare alors la tendance moderne à libéraliser les choix à l'habitude qu'avaient certains peuples nomades de se débarrasser des individus faibles, malades ou trop vieux. Or cette comparaison n'a pas de pertinence. La motivation de ces peuples nomades n'était aucunement rattachée à un quelconque respect des choix individuels. Au contraire il s'agissait de sacrifier l'individu à la subsistance du groupe. L'euthanasie qui est pratiquée dans les sociétés modernes l'est par compassion et par respect des individus. C'est justement parce que l'intérêt de subsister, pour le groupe, est mis en sourdine qu'on accepte la mort douce d'un proche. La tendance de la sensibilité moderne, au contraire de celle de ces peuples nomades, est plutôt de cesser de subsister que de sacrifier un seul des siens contre son gré. Là réside la différence de l'attitude éthique nouvellement apparue avec la modernité. L'idée même de sacrifier expressément un individu pour la subsistance de la famille ou de la communauté est devenue intolérable. L'ancienne normalité des nomades est ressentie là comme une absurdité.

- La mise en question moderne du caractère absolu de la vie s'est accompagnée d'une nouvelle sensibilité morale à l'égard de l'autonomie individuelle. C'est ce qui fait qu'elle n'est pas une régression vers des attitudes archaïques ou barbares. Cellesci dénotent bien un faible respect de la vie individuelle, il est vrai, mais elles témoignent bien plus d'une totale absence de considération pour l'autonomie individuelle, la reconnaissance de la personne et de ses choix propres.

II s'agit pour nous de comprendre la raison profonde d'un certain nombre de faits. D'après ce qui précède, nous voyons 
qu'il persiste une fausse évidence quant au caractère sacré de la vie, lequel est présenté encore de nos jours comme devant être admis sans discussion. D'où la position précaire des tenants de l'autonomie individuelle dans les questions éthiques où la vie est en cause. Une fausse perspective historique quant à la signification de la modernité alimente la confusion. Or, pour rétablir la perspective, il s'agira précisément de comprendre le pourquoi de toute cette confusion et de renverser l'évidence en faveur de l'autonomie individuelle, principe de base dont l'éthique, selon nous, ne devrait pas s'éloigner pour quelqu'idole que ce soit.

\section{Correspondance avec le paternalisme classique.}

Dans les questions de morale, il semble bien d'abord que l'argument du respect de la vie a été utilisé d'une façon plus marquée dans la modernité, surtout au vingtième siècle. La vie humaine avait-elle moins de prix aux époques antérieures, estce qu'on s'entretuait plus facilement? Ou alors est-ce surtout une question de sensibilité, la sensibilité moderne étant plus susceptible de donner lieu à des protestations contre la violence meurtrière en général? Quoi qu'il en soit, c'est un fait que, dans les débats modernes, l'argument du respect de la vie est fréquemment employé et qu'il est considéré sérieusement. Toute l'argumentation des opposants à la libéralisation dans les questions de bioéthique en fait un pivot. Nous voulons montrer ici qu'un autre type d'argumentation a été utilisé pour les conflits moraux des siècles passés - notamment à l'époque classique, soit autour des $X V I^{\theta}$ et $X V I I^{e}$ siècles - et qu'il a joué un rôle également important dans un contexte de conceptions qui peut être mis en correspondance d'une façon frappante avec le contexte de l'argumentation qui se tient autour des principaux conflits moraux de la modernité. II s'agit de l'argumentation classique qui est basée sur le principe de l'autorité paternelle. Comme dans le cas de la "vie» à l'époque moderne, le "père» est une notion qui, à l'époque classique particulièrement, a servi de pivot à une argumentation de fond contre certaines formes de révoltes subjectives et qui renvoyait à une conception quasi religieuse, où le sacré et l'absolu prenaient leur part. Le «père», 
comme la "vie", se réfèrent à des réalités qui n'ont rien de sacré au départ. Ce sont des réalités ordinaires, naturelles, banales. Ces notions ont cependant été sacralisées en étant appuyées sur une réalité d'ordre spirituel qui les transcendait.

Dans la modernité, le caractère absolu de l'autorité paternelle est largement tombé. Notre approche permettra de voir que, dans les conceptions de fait, le caractère absolu de la vie s'est introduit dans l'espace alors vacant, espace déplacé par rapport à l'ensemble des conceptions des époques respectives. Il ne s'agit pas ici proprement d'analogies, mais de correspondances conceptuelles qui engagent des ensembles de conceptions très larges et qui couvrent toute une époque de la pensée. Des analogies se soutiendraient par quelques points de ressemblance. Ces correspondances s'établissent au contraire entre des conceptions qui n'ont, le plus souvent, rien de semblable.

\section{L'autorité paternelle classique.}

Le mot "père" a connoté fortement l'autorité dans les sociétés occidentales à certaines époques. Pour le moderne, cetteconnotation est dépassée. Elle apparaîtsouventaujourd'hui comme le paradigme de l'esprit traditionaliste.

Nous nous intéresserons spécifiquement à la signification classique du "père», celle qui remonte au moins aux XVII" et $\mathrm{XVIII}{ }^{\theta}$ siècles. Voici comment Daniel Rops décrit le pèrë au sens classique du terme :

Le père de famille, chef responsable de la cellule sociale, a une autorité qui, à son rang, rappelle celle du roi dans le royaume, et qui, comme la sienne, est d'essence religieuse. Le respect dont il est entouré, les pouvoirs qui lui sont reconnus, sont presque inconcevables dans la société moderne, imprégnée d'idées égalitaires. Ses droits testamentaires, beaucoup plus étendus que de nos jours, manifestent cette autorité par delà la mort...

7. D. Rops, L'Église des Temps classiques, Le Grand Siècle des Âmes, Paris, Arthème-Fayard, 1958, p.277 
On imagine encore assez bien la famille traditionnelle, où le père siégeait au bout de la table du côté du feu, et où sa femme s'occupait à servir les plats. II est bien connu que les parents avaient le droit effectif et moral de déshériter leurs enfants si ceux-ci agissaient contre leur volonté, notamment s'ils se mariaient sans leur consentement. On a cependant peut-être oublié à quel point la fonction paternelle était privilégiée et sacrée, à une époque où l'on considérait comme parfaitement sain et normal ce qu'un moderne verrait comme de détestables abus de pouvoirs.

Au XVIIe siècle, les conceptions de l'époque suggéraient l'argument suivant et le faisaient péremptoire : l'enfant doit tout à son père puisqu'il lui doit la vie. La puissance paternelle s'étendait donc sur la personne et les biens des enfants, petitsenfants et autres descendants. On admettait comme une évidence que l'enfant, sujet de l'action de ses parents, n'avait d'autres droits ou devoirs que de les écouter et de leur obéir. Le pouvoir du père sur sa famille était absolu et perpétuel. Les dispositions testamentaires assuraient son prolongement, même après le décès du père. Ces contraintes avaient pourtant une contrepartie : la bonté associée normalement au rôle du père.

Ainsi concevait-on l'autorité paternelle à l'époque classique. Sacrée et inexorable, elle était la condition de tout accomplissement réel, de toute "vraie liberté". Toute rébellion, toute remise en question étaient a priori condamnables et ne méritaient absolument aucun égard.

\section{Conflits classiques et conflits modernes.}

François Sarda qualifie les conflits sociaux sur l'euthanasie et l'avortement de "débats étranges". II trouve étrange, en effet, que s'y croisent le cœur contre la raison, les faits contre les principes ${ }^{8}$. Or, c'est dans ces mêmes mots que l'on décrirait le mieux la nature des fameux conflits classiques que les grands dramaturges ont mis en scène. Certes, les questions visées par 
Sarda ne sont aucunement traitées dans le théâtre classique. Ce sont des questions spécifiquement modernes, alors que le théâtre classique regorge plutôt de dilemmes où le rapport père-fils est en jeu. Cependant, notre correspondance entre l'autorité paternelle et la qualité intouchable de la vie peut d'abord s'alimenter à cette comparaison. D'une part, le principe du respect de la vie est central dans nos codes de justice et de morale. D'autre part, le rôle du père de famille classique servait logiquement de support à l'ensemble des conceptions morales.

Ainsi, comme exemple préalable de ce procédé, prenons le cas de remarques de l'historien Jean-Louis Flandrin. Celui-ci considère comme un paradoxe la situation suivante. Beaucoup de chrétiens de nos jours refusent l'esclavage, la monarchie, la direction paternaliste des entreprises et mettent même en question les pouvoirs des parents sur les enfants. Toutefois, ils continuent de croire en un Dieu qui se présente comme un Père créateur et qui agit selon ses propres desseins souvent impénétrables ${ }^{9}$. II y a bien là une contradiction entre les prises de position sur des matières concrètes et une croyance en un absolu. Ce type de contradiction, qui est un rapport entre termes appartenant à un ensemble de conceptions, existe également chez ceux qui prennent position sur les questions où le respect de la vie est en cause. Il est en effet très fréquent de voir que ceux qui défendent le droit à l'avortement et à l'euthanasie continuent de parler de la vie comme de quelque chose qui est sacré. Ils ne se rendent apparemment pas davantage compte de la contradiction de leurs positions que ceux qui ont été mentionnés ci-dessus ne le font eux-mêmes.

Flandrin, par ailleurs, fait d'autres remarques qui nous importent ici. Il constate que, du début du $X V^{\ominus}$ siècle au début du XVIII ${ }^{e}$ siècle, l'intérêt du clergé pour la vie domestique s'est continuellement accru ${ }^{10}$. Nous pouvons observer, corrélativement, que l'intérêt de l'Église pour les questions où le respect

9. J.-L. Flandrin, Familles, parenté, maison, sexualité dans l'ancienne société, Paris, Seuil, 1984, p. 118.

10. Idem, p. 120. 
de la vie est en cause s'est accentué singulièrement depuis le $\mathrm{XIX}^{\mathrm{e}}$ siècle jusqu'à nos jours. Ces faits ont une pertinence pour l'établissement de nos correspondances conceptuelles. Ainsi, on constate d'une part que les Églises chrétiennes ont fait de la famille un des lieux privilégiés de la vie religieuse. Cela s'explique par les possibilités offertes pour surveiller et éduquer la masse des fidèles. En corrélation, d'autre part, on constate que les questions où le respect de la vie est en cause sont importantes à considérer pour pouvoir critiquer et tenter d'exercer un contrôle sur les nouvelles techniques médicales ou autres à partir desquelles s'érigent en grande partie les états modernes.

Notons ici que ce n'est pas proprement l'Église telle qu'elle existe aujourd'hui qui doit être mise en correspondance conceptuelle avec l'Église classique. Ce sont plutôt l'ensemble des agents critiques qui participent à la mise en place des principes des jugements pour toutes les questions qui concernent les sociétés modernes et les rapports entre les sociétés. Ce sont donc, par exemple, l'ensemble des communautés instruites en matière de sciences humaines et de questions d'éthique, les chercheurs, les auteurs, les enseignants. Ces communautés, ou ces écoles, jouent aujourd'hui un rôle que l'on peut mettre en correspondance conceptuelle avec celui des théologiens et des moralistes de jadis.

\section{L'argument de la vie comme fondement de l'humain.}

Deux des arguments les plus forts - ou du moins perçus comme tels par les tenants du respect absolu de la vie - sont l'argument de la vie posée comme fondement de la réalité humaine et l'argument du glissement, selon lequel un quelconque relâchement vis-à-vis du principe du caractère sacré de la vie entraînerait, à plus ou moins long terme, une catastrophe générale, soit la perte complète de toute valeur accordée à la vie humaine. Analysons d'abord le premier de ces deux arguments. 
Cet argument peut être formulé ainsi :

II ne peut clairement pas y avoir de vie humaine personnelle, de vie capable de jouissance, d'entrée en relation, de communication, et ainsi de suite, à moins qu'il n'y ait aussi une vie biologique humaine ${ }^{11}$.

Tel quel, cet argument se conçoit comme un truisme. C'est l'évidence même. Pourtant il recèle une faute logique qui devrait normalement sauter aux yeux de tout observateur un peu au fait des distinctions philosophiques touchant les notions de vie biologique, de vie personnelle, de communication, etc.

Lorsqu'un argumentest considéré de façon générale comme fort, qu'il est très utilisé et que, de plus, il est avancé comme une vérité péremptoire, il y a bien des chances qu'il ne soit que l'expression d'un préjugé régnant ou d'une idée reçue. Seul un recul suffisant peut faire sentir son caractère illusoire. Pour faire comprendre ce point, envisageons le correspondant conceptuel de cet argument-truisme, portant sur les rapports du père et du fils tels que conçus à l'époque classique.

Afin d'effectuer la transposition, notons d'abord que l'argument en question est motivé généralement par un désir de répondre à des opposants qui prétendent mettre de l'avant le caractère essentiel des choix personnels, de la communication, etc. Alors on fait simplement observer que les choix personnels et la communication, etc., présupposent bien évidement la vie biologique.

Le cas classique des rapports du fils au père se présente comme suit. On peut faire valoir que le fils possède un jugement personnel, autonome. Le penseur classique, moraliste, philosophe ou théologien, a beau jeu, pense-t-il, de rétorquer: tout jugement personnel ou autonome du fils ne peut exister que parce qu'il lui a été transmis par son père et l'éducation que celui-ci lui a prodiguée. Au regard classique, cet énoncé paraît tellement juste que c'est un truisme. Le moderne le voit tout autrement. D'abord, il entend autre chose par les mots «person- 
nel» ou «autonome». Il serait absurde aujourd'hui de prétendre que l'autonomie ne peut exister que par l'obéissance à un quelconque tuteur. En outre, on admet généralement le rôle essentiel de l'ensemble des rapports sociaux dans la formation individuelle. On ne croit plus au rôle suprême et quasi exclusif des parents, a fortiori du père.

\section{L'interdit de l'homicide comme condition de possibilité du dialogue.}

L'argument examiné ci-dessus peut être présenté pour l'essentiel selon une interprétation différente. Jean-François Malherbe l'exprime comme ceci :

La première condition de possibilité du dialogue sera [...] de respecter l'interdit de l'homicide ${ }^{12}$

Afin de pouvoir procéder à notre analyse conceptuelle, nous interpréterons cet énoncé de façon quelque peu libre vis-à-vis des intentions de son auteur. L'énoncé, pris en lui-même, peut être interprété en un sens étroit ou en un sens large. Dans le premier cas, il est tautologique, c'est-à-dire vide de contenu; dans le second cas, il est contradictoire. Dans le sens étroit, il désigne le respect de la vie de l'interlocuteur lui-même dans le dialogue, ce qui n'est qu'une conséquence immédiate du respect de l'interlocuteur en tant que tel. Dans le sens large, il signifie l'interdiction de l'homicide en général comme condition de possibilité du dialogue, ce qui est contradictoire puisque le dialogue peut précisément porter, en principe, sur l'établissement des limites du respect de la vie en général.

Ces difficultés conceptuelles peuvent paraître considérables aux yeux de modernes qui sont aux prises avec les graves questions de l'euthanasie ou de l'avortement. Considérons cependant l'argument classique correspondant et constatons que les difficultés sont plutôt spécieuses. Tout d'abord, il conviendra de trouver le correspondant conceptuel classique

12. J.-F. Malherbe, Pour une éthique de la médecine, Paris, Larousse, 1987, p. 45. Selon cet auteur, il faut comprendre cette condition sous l'aspect de la cause formelle, en référence à la typologie aristotélicienne. 
du dialogue au sens moderne. Celui-ci joue un rôle essentiel dans les rapports sociaux, si du moins on l'entend dans le sens de l'élément de base de la communication. Le correspondant classique doit être cherché, conformément au sens initialement donné aux correspondances conceptuelles, dans les rapports intra-individuels. Alors nous pouvons voir que la réflexion personnelle, et plus particulièrement la mémoire, seront substituées ici au dialogue. Nous obtenons l'argument :

La première condition de la mémoire est de respecter l'autorité paternelle.

Certes, ainsi formulée, cette proposition est bizarre. Elle ne l'est pas vraiment plus que l'énoncé de Malherbe pris en luimême. Au sens étroit, elle signifie que la mémoire du fils ne peut que tenir compte de l'existence du père, ce qui est sans contenu intéressant. Au sens large, elle signifie que la mémoire individuelle n'est possible que si l'individu obéit à son père, ce qui est manifestement faux. Toutefois ce qui saute aux yeux d'un moderne dans ce cas apparaîtrait beaucoup moins évident aux yeux d'un moraliste classique, qui ne verrait dans cette critique, sans doute, qu'une vaine argutie.

En fait, le regard moderne se transforme en une critique de fond si l'on considère la situation selon la perspective du freudisme par exemple. Celle-cia même pour effet de renverser les conclusions de l'argument présenté ci-dessus. Non seulement il n'est pas nécessaire de respecter l'autorité paternelle pour se souvenir, mais en outre les manifestations plus ou moins conscientes de cette autorité - sous la forme du surmoi notamment - nuisent à un travail de remémoration personnel. C'est toute la question du refoulement et des névroses qui est en cause. L'anamnèse au sens psychanalytique consiste précisément à se remémorer, par delà les refoulements et de façon à surmonter le sentiment de culpabilité lié aux effets de l'autorité paternelle.

II se faitjour la possibilité d'interpréterl'argument de Malherbe selon une ligne conceptuelle qui se trouve en correspondance avec la critique et le dépassement freudiens. Non seulement 
l'interdit de l'homicide n'est pas une condition de possibilité du dialogue, mais il constitue une négation du dialogue possible. Cela est vrai, d'une part, à cause des effets culpabilisants, donc entravants, de cet interdit, surtout s'il est interprété de façon restrictive, et d'autre part, vu les conditions d'accomplissement du dialogue effectif. II suffit de penser - de façon quelque peu schématique il est vrai - que le dialogue suppose un minimum de quiétude et de sérénité qui, dans certains cas, ne peut être assuré que par des moyens de défense légitimes contre les nuisances. Dans le cas particulier d'un dialogue dont certains participants sont des partisans du droit à l'euthanasie ou à l'avortement libres, on peut même identifier ces nuisances aux comportements de tenants trop absolutistes du respect de la vie. Cette nuisance au dialogue moderne, en tant qu'échange social historiquement situé, s'apparente à une forme de refoulement qui s'opère au sein de la société elle-même.

\section{L'argument du glissement.}

Second argument le plus fort, peut-être, l'argument du glissement consiste à présenter comme probable une grave diminution générale du respect de la vie, si la moindre brèche y est ouverte au départ. Parfois, on va plus loin et on avertit que cette brèche, si elle était permise, serait une voie ouverte à tous les crimes. Le même argument du "glissement» s'applique aussi bien à la situation classique, à la condition de faire la correspondance de façon appropriée. La façon absolutiste de considérer le respect de la vie est en correspondance avec l'attitude classique ferme et intransigeante soutenant l'autorité paternelle. Celle-ci est alors vue comme le modèle et le représentant de toute autorité. Si l'autorité paternelle n'est pas parfaitement respectée, c'est non seulement le père qui se trouve bafoué dans sa responsabilité et son devoir, mais c'est toute la notion d'autorité qui est menacée.

Par extension, l'argument du glissement consiste à prendre une position restrictive dans les questions d'euthanasie, pour la raison que des abus sont possibles. Par exemple, on prétend qu'il faut interdire l'acte individuel d'euthanasie parce que les 
motifs ou les inspirations de ceux qui aident à mourir ne sont pas toujours nobles. Le schéma est classique en définitive et on le reconnaît bien dans les cas de condamnation du fils par le père. La désobéissance du fils sera qualifiée d'anarchique même si elle résulte d'un acte d'autonomie et d'une réflexion sérieuse. On ne veut pas tolérer les écarts de conduite car ils sont le prodrome de la révolte; ils sont de mauvais exemples et ils nuisent à l'image d'autorité du père, donc à l'exercice de son autorité. Cela s'entend, dans une société hiérarchisée de façon rigide. Toutefois ceux qui, à l'époque moderne encore, s'en tiennent à ce schéma classique des relations du père et du fils sont qualifiés de traditionalistes encroûtés. Leurs craintes, considérées naguère comme légitimes, ne paraissent plus fondées et font figure d'obsessions morbides. Le fils peut parfois bafouer son père. Ce doit être jugé comme un cas général de manque de respect d'autrui. On n'a pas à invoquer l'argument du glissement qui fait conclure au danger d'anarchie totale. En correspondance, on verrait que l'intention parfois mauvaise de celui qui aide à mourir doit être jugée comme un cas quelconque d'abus vis-à-vis de l'intégrité physique ou psychologique d'une personne. Ce n'est pas l'euthanasie qui doit être dénoncée, mais bien l'atteinte générale à autrui, à son intégrité et à sa liberté.

\section{L'argumentation autour de l'avortement.}

L'argumentation moderne possède des traits spécifiques qui la distinguent de certaines argumentations anciennes. Parmi ces dernières celle de l'Église catholique a des bases et une sensibilité différentes. Mais il est intéressant de comparer les conceptions modernes avec un point de vue antique non judéo-chrétien. Tacite considérait les Hébreux comme un peuple barbare, parce qu'ils n'avaient pas l'attitude rationnelle de supprimer les enfants qui étaient de trop. Voilà un argument que peu de modernes reprendraient tel quel. II suppose en effet cette froide «rationalité» qui consiste à tuer par utilité sociale ou autre. Des auteurs modernes auraient tôt fait, devant un tel argument, de le rejeter en évoquant le spectre du nazisme par 
exemple. En ce sens, quand l'Église catholique s'oppose à l'expression d' "avortement thérapeutique" pour la raison que l'avortement doit toujours être un aveu d'échec, elle prend ses distances vis-à-vis de la rationalité utilitaire et se rapproche par le fait même d'une sensibilité plus répandue à notre époque que dans l'antiquité de Tacite.

La sensibilité moderne, cependant, s'éloigne de celle qui anime la morale catholique traditionnelle quand on considère les mobiles des personnes qui souhaitent un avortement. Elle perçoit ces mobiles comme dignes et honnêtes, contrairement à la morale traditionnelle qui les voit toujours comme sordides. On admet ainsi ces nouvelles raisons, qui s'intègrent dans une conception d'ensemble des personnes des deux sexes : la libéralisation de l'avortement permet d'éliminer l'avortement clandestin, favorise l'épanouissement de la femme et rend possible une plus grande égalité de fait entre les hommes et les femmes. Nous sommes loin, ici, de la rationalité à la Tacite, puisque celui-ci n'envisageait certainement pas l'utilité de l'avortement dans le sens de l'épanouissement égalitaire de toute personne. On peut affirmer que la sensibilité moderne est, au moins sur ce point, davantage éloignée de celle de Tacite que ne l'est la sensibilité de la morale chrétienne traditionnelle.

D'autres considérations, non directement morales, font partie de la figure d'argumentation. Elles paraissent déterminantes la plupart du temps dans les faits. On remarque que la libéralisation de l'avortement va souvent, dans les différents pays du monde, dans le sens inverse des craintes démographiques. C'est probablement plus vrai pour des pays non occidentaux, comme l'Inde ou la Chine. On allègue que la raison d'État et les considérations sur la fertilité de la nation l'emportent sur les débats qui impliquent le respect de la vie de la personne. Ce point de vue, au-delà de ce qu'il peut présenter de superficiel ou de simplement pragmatique, se trouve en correspondance avec un point de vue classique sur l'obéissance filiale. Le fils ou la fille ont, en effet, une "bonne raison" de ne pas se rebeller contre une décision du père : ils risquent d'être déshérités. Ces 
deux points de vue mis ici en correspondance, liantl'importance des raisons d'ordre matériel (ou économique) sous-jacentes à certaines positions morales, sont des arguments supplémentaires à la disposition des critiques de l'ordre classique ou de la stabilité au sens moderne. Ils mettent en fait le doigt sur un des traits structuraux qui semblent maintenir le système établi. Cependant celui-ci fait corps aussi bien avec l'ensemble des conceptions morales, sans lesquelles il perdrait son sens ou sa spécificité par rapportà d'autres ensembles conceptuels. Autrement dit, la figure générale et intouchable du Père ou de la Vie caractérise davantage la structure conceptuelle que tel ou tel trait de fonctionnement objectif, qui ne détermine rien de façon univoque dans les formes conceptuelles de l'ensemble d'une époque. C'est d'ailleurs ce que démontre l'examen de la figure d'ensemble lorsque nous élargissons l'argumentation de façon à englober plusieurs autres problèmes que ceux qui concernent l'avortement.

\section{Refus de la vie et désobéissance filiale.}

II ne s'agit pas de réfuter un argument puis un autre, mais plutôt de comprendre comment une conception globale en remplace une autre. De plus, il n'est pas question de comparer le traitement classique au traitement moderne réservé au suicide. La situation classique qui se trouve en correspondance conceptuelle avec la question moderne du droit à la mort est celle qui concerne le droit filial de dissidence vis-à-vis de l'autorité paternelle.

La situation classique du rapport père-fils comporte un certain nombre d'aspects essentiels qui font corps avec l'ensemble de la conception classique de l'autorité. Et ce sont les critiques effectuées par la suite qui nous importent, car elles présentent une originalité de pensée qu'il nous faut reproduire à un tout autre niveau.

Ainsi on a reconnu après coup que le fils qui désobéit ne remet pas généralement en question l'autorité paternelle ellemême, mais plutôt la manière dont elle est exercée. C'est le 
père qui, souvent, a perdu son autorité, bien plus que le fils qui l'a bafouée.

Le père abusif qui a perdu son autorité aux yeux de son fils représente une situation significative. Dans ce cas on dit volontiers que le fils désobéissant ne s'attaque pas à l'autorité elle-même mais à son mauvais exercice. Or l'autorité de la société classique garantissait continuellementl'autorité du père. Cependant, le fils rejette bel et bien l'autorité du père. Pour lui, quelque chose vient de se rompre à ce niveau du réel et, à travers la figure incompétente de son père, il en vient à rejeter non seulement une réalité particulière, mais le droit même de son père à exercer une autorité sur lui.

Nous retrouvons cette formation conceptuelle - un certain type de rapport père-fils - à un autre niveau, et complètement transformée. L'homme déçu par la vie et qui décide d'y mettre fin ne pose généralement pas, dit-on, un verdict négatif sur la valeur de l'existence elle-même. Ce n'est pas la Vie qu'il vise mais la forme qu'elle a prise pour lui. C'est une objection importante contre l'admissibilité morale du suicide, dans la mesure où l'on suppose la possibilité effective de changer de vie. Cependant l'homme qui a renoncé à la vie de façon libre et volontaire trouvera cette objection superficielle et vide. II se méfie de tout mirage apaisant dont l'acceptation serait une trahison de ce qu'il a enfin décidé de son être et pour son être. C'est en fin de compte beaucoup plus qu'une vie particulière qu'il rejette: soit le jeu intégral auquel cette vie particulière participe. La vie décevante est en correspondance conceptuelle avec le père abusif. Dans les deux cas, c'est toute une figure du bien en soi qui est flétrie et qui tombe.

On a parlé de pharisaïsme de la société actuelle vis-à-vis de l'aide au suicide ${ }^{13}$. Elle se montre en effet d'une grande sévérité envers celui qui aide au suicide alors que le suicide lui-même n'est pourtant plus un crime inscrit dans la loi. Cette situation

13. Voir par exemple Raymond Lemieux, "Suicide et société", et Willy Apollon, "Enjeux éthiques de la mort volontaire", dans Les Suicides, op. cit., p. 144 sqq. 
correspond à la distinction faite classiquement entre le blasphème et la mauvaise pensée. Le premier était passible de lourdes peines (jusqu'à la peine de mort) alors que la seconde ne s'attirait qu'un blâme théorique. On dirait qu'une situation de fait commande l'attitude morale. Dans l'horizon classique, le blasphème fait figure d'exemple pernicieux dont est responsable l'instance individuelle qui en entretient l'habitude. L'aide au suicide apparaît, dans l'horizon moderne, comme une forme de comportement dangereux en raison du glissement qu'il suggère vers des abus motivés par des intérêts sordides.

Il existe une correspondance des critiques. On en sera venu à voir dans le blasphème une faute légère, voire une expression de sincérité et une forme de sérieux et, dans certains cas, de courage. De même, celui qui aide au suicide s'expose plus qu'il ne profite. S'il y a faute, elle ne peut être bien plus grave que celle du suicide lui-même (comme le blasphème n'est pas en soi un bien plus grand péché que la mauvaise pensée). On y verra de plus en plus, sans doute, un geste d'altruisme et de conviction. La société assez juste pour ne pas sanctionner l'aide au suicide sera elle-même considérée, toujours par correspondance conceptuelle, comme se conformant à une éthique plus décentrée et plus authentique.

Une autre figure classique du rapport père-fils est celle qui fait voir la révolte décidée du fils contre le père comme étant la manifestation d'une des pires malices. La haine intériorisée par le fils est alors plus grave qu'une atteinte particulière à l'intégrité physique du père. Le fils qui frappe son père peut encore être réchappé moyennant une amende honorable et un châtiment exemplaire. Mais le fils rebelle n'aura aucune chance de salut.

Le suicide, pour sa part, a été longtemps considéré comme étant au moins aussi grave que le meurtre, même si la menace apparente pour la société est moins évidente. Le meurtrier tue en général tout en continuant de croire à la vie. Le suicidant pose un acte qui consiste à maudire la vie. Là est la révolte. Au $\mathrm{XX} \mathrm{X}^{\ominus}$ siècle, on en est arrivé à sanctionner moins le suicide que le meurtre. Du point de vue éthique, la charge réprobatrice demeure très élevée vis-à-vis du suicide. Qu'en est-il du danger 
social lié au suicide, c'est-à-dire de la perception que la modernité en a? On y a vu la plupart du temps un mal social profond, un risque d'épidémie.

Selon Camille Schuwer, le suicide est inoffensif parce qu'il est inimitable. Seul le suicide événement, selon lui, peut être un exemple qui risque d'être suivi. Or le suicide tend à demeurer caché $^{14}$. Cette conclusion de Schuwer est certes discutable. Le suicide ne peut rester généralement soustrait de la conscience sociale même si, dans plusieurs cas, l'entourage du suicidé préfère garder le secret. Le suicide est imitable jusque dans l'exemple même de la liberté en acte. Schuwer a pourtant raison de dire qu'il est inoffensif, mais c'est plutôt parce que le suicide n'est pas essentiellement une offense contre qui que ce soit. Cette liberté n'est pas mauvaise en soi. Elle est perçue comme étant mauvaise par ceux qui craignent les effets plus ou moins réels de la liberté, par ceux qui comprennent mal l'altérité des raisons. Cette configuration conceptuelle en éthique correspond à celle qui concerne le fils indigné. L'indignation intérieure en tant que telle est inoffensive par définition. Elle peut devenir menaçante à l'égard d'un père contre qui le fils entretient un sentiment d'irritation. On ne peut nier que le fils, un jour excédé, puisse en arriver à brutaliser son père. Cette possibilité est inscrite dans l'ordre même du rapport père-fils. L'indignation du fils ne devient mauvaise que lorsqu'elle se mue en révolte ouverte.

Un renversement de perspective quasi fantastique s'opère en passant du regard classique au regard moderne à propos du rapport père-fils. Non seulement on ne reconnaît plus l'autorité paternelle dans son aspect imprescriptible et discrétionnaire, mais en outre, on ne peut même plus comprendre de l'intérieur la sensibilité morale dont elle procédait. La critique moderne a été dévastatrice. On considère maintenant comme allant de soi qu'il faut plutôt respecter l'homme que le père en tant que tel. II est naturel et humain tout à la fois de s'opposer à toute forme

14. C. Schurver, La Signification métaphysique du suicide, Paris, Aubier, éd. Montaigne, 1949, p. 146. 
d'autorité abusive. La modernité en fait même souvent une sorte de devoir. C'est une question de pragmatisme sans doute que de ne pas obéir aveuglément. C'est aussi au nom de la vérité et de la liberté que l'individu doit refuser la domination d'autrui, particulièrement quand elle est cautionnée par la société existante. Le fils qui se révolte, au lieu d'être la figure du mal, apparaît comme plutôt franc, direct et courageux. II représente l'humain au meilleur sens du terme.

Dans la configuration conceptuelle globale, le renversement de perspective qui équivaut au passage d'un ordre d'horizon à un autre ordre d'horizon est une figure essentielle. Un autre élargissement de l'ordre conceptuel, qui amènera un changement d'horizon par le fait même, aura des effets aussi profonds et aussi formidables. Pour bien en apprécier la signification, notamment sur le plan éthique, il faut tenir compte d'un changement de sensibilité radical. Alors, il est prévisible que le respect du caractère sacré de la vie tombera complètement. On critiquera dans la Vie ce qu'elle a d'aveugle, d'inhumain ou d'abusif pour l'instance subjective qui subit. On réalisera par exemple plus clairement que le système de la vie est un système de violence et de rapports de force où les êtres sont toujours en lutte les uns contre les autres. On en viendra à discréditer la vie au profit du désir. C'est-à-dire que celle-là ne vaut que par celuici, tout comme on en est arrivé à considérer que le respect dû au père passe par sa qualité d'homme. Une vie sans désir, une vie non désirée, n'ont pas plus de valeur que l'autorité d'un père abusif. L'homme qui décide de mettre fin à sa vie apparaîtra comme une figure respectable de la liberté. On décapera une sensibilité morale devenue insuffisante et superficielle.

Comme l'ancien droit du père à son pouvoir et à son rôle autoritaire découle d'une situation de fait, alors que le droit du fils à son autonomie est beaucoup plus essentiel, on admettra également que la liberté de vivre ou de mourir est fondamentale au regard du respect dû à la vie. 


\section{Suicide et blasphème.}

Le blasphème était un crime grave à l'époque classique. Un des premiers gestes de Louis XIV, après qu'il eut commencé à régner, fut de réitérer un édit punissant de mort les blasphémateurs. Le blasphème, ou parole impie et outrageante, se situe entre la prière vigoureuse d'une part, et le sacrilège, l'action profanatrice, d'autre part. L'action suicidaire, qui est également perçue comme manquant gravement de respect envers une instance supérieure, correspond au blasphème. Elle se situe quelque part entre le sacrifice pour une cause valorisante (celle du martyr, du soldat, du sportif...) et le meurtre. Le blasphème est trop brutalement égocentrique pour être une prière, qui suppose une adresse à quelqu'un et un échange. Le suicide ne se fait pas pour une cause reconnaissable socialement.

L'acte suicidaire apparaît comme une reprise conceptuelle de la prière classique, prise en un sens particulier. Les observateurs modernes parlent du suicide, ou de la tentative de suicide, comme d'un symptôme, d'une communication, d'un signe qui fait partie d'un dialogue ${ }^{15}$. L'acte suicidaire n'est pas seulement en correspondance avec le blasphème. II lui ressemble par son aspect radical et imprévu. Il déstabilise l'entourage et la conduite suicidaire se caractérise par la récidive, parfois par l'escalade. L'acte suicidaire, dit-on, est sauvage. Toutes ces caractérisations traduisent en même temps la fébrilité du corps social devant un phénomène interne qu'il ne parvient pas à assumer. L'acte suicidaire, comme jadis le blasphème, est l'irruption dénonciatrice de l'intolérable qui est elle-même intolérable. L'examen des formes conceptuelles nous apprend que ce type de problème, d'une infinie gravité à l'intérieur du cercle d'un horizon donné, n'est finalement surmonté, mais alors il l'est complètement, que lorsqu'on passe au-delà de cet horizon. 
Et on comprendra que les craintes apparemment justifiées de la société moderne vis-à-vis d'un phénomène de liberté individuelle si terrible ne reposent que sur l'évaluation factice des risques de déstabilisation. Cette évaluation est inhérente à I'horizon particulier des conceptions. II en allait entièrement de même dans le cadre classique : la désobéissance filiale était un mal absolu car elle paraissait dévastatrice pour l'ensemble de la structure. Un élargissement cependant, c'est-à-dire un dépassement de cet horizon, et il est apparu au contraire que le phénomène "dévastateur" était plutôt sain et normal. Les rapports entre individus ne s'en portent que mieux depuis que l'ancien rapport père-fils est tombé en désuétude. Le respect voué à l'homme-père n'en est que plus réel et plus solide. II en ira de même, par correspondance, pour le respect dû à la viedésirée: il en sera plus authentique. Toutes les craintes de déstabilisation à cet égard se seront évanouies par le fait même.

Yvon Provençal Département de philosophie Collège de Granby 


\section{Bibliographie sommaire.}

Barnard, C., Choisir sa vie, choisir sa mort, trad. H. Denès, Éditions Pierre Belfond, Paris, 1981.

Bélanger, R., Les Suicides (ouvrage collectif), Editions Fides, Montréal, 1985.

Bénichou, P., Morales du grand siècle, sixième édition, Paris, 1948.

Camus, A., et Koestler, A., Réflexions sur la peine capitale, Calmann-Lévy, Paris, 1957, 1979.

Cragg, W., Contemporary Moral Issues, McGraw-Hill Ryerson Lted, 1987.

Dubarle, D. et Dessus, G., «Le prix d'une vie humaine», Cahiers Laënnec, Paris, juin 1963.

Dumas, A., Problèmes éthiques de l'avortement (ouvrage collectif) éditeur Édouard Privat, Toulouse, 1973. 